
input and output coordinates and velocities of every individual muon that passed through the experimental apparatus. As a result, the authors could unequivocally demonstrate that they had achieved ionization cooling of muons.

Organizations worldwide are developing long-term strategies for exploring the high-energy frontier. Plans include designs for circular colliders up to $100 \mathrm{~km}$ in circumference and linear colliders up to $50 \mathrm{~km} \mathrm{long}^{7}$. Although these approaches, which would use protons or electrons and positrons, have the least technical risk, they still have a substantial cost, as well as technical challenges, that affect their feasibility.

Other plans include designs that would use innovative technologies such as those based on lasers and plasmas ${ }^{8}$. These approaches have made great progress in developing compact accelerator stages at low energy, but the combined use of such stages to reach high energies while retaining a high beam quality will require many years of research and development. Still other plans involve muon beams 9 .

Thanks to the MICE collaboration, the first demonstration of ionization cooling of muons has been achieved. However, it must be noted that the amount of cooling was small. Although conceptual designs for muon colliders have been developed ${ }^{9}$, establishing the viability of a realistic muon-cooling system and of a muon collider will need much more work.

It is too soon to say which, if any, of the proposed approaches will provide a technically and financially feasible path to the future energy frontier. But if physicists can learn how to cool and control muon beams, then it is hard to imagine that putting muons in a circular collider will not be the way forward. These particles offer clean collisions (unlike protons) and lose little energy when their trajectories are bent by accelerator magnets (unlike electrons). As a result, a muon collider could reach energies that match or surpass those of an electron or proton collider, but be substantially smaller. The MICE collaboration's work is a milestone on the road to realistic muon-cooling systems that could someday lead to neutrino factories and muon colliders.

Robert D. Ryne is in the Accelerator Technology and Applied Physics Division, Lawrence Berkeley National Laboratory, Berkeley, California 94720, USA.

e-mail: rdryne@lbl.gov

MICE collaboration. Nature 578, 53-59 (2020)

2. Panofsky, W. K. H. \& Breidenbach, M. Rev. Mod. Phys. 71 S121-S132 (1999).

European Strategy for Particle Physics Preparatory Group Preprint at https://arxiv.org/abs/1910.11775 (2019).

4. Rubbia, C. Int. J. Mod. Phys. A 33, 1844010 (2018).

5. Skrinsky, A. N. \& Parkhomchuk, V. V. Sov. J. Part. Nucl. 12 223-247 (1981).

6. Neuffer, D. Part. Accel. 14, 75-90 (1983).

7. Amaldi, U. et al. Preprint at https://arxiv.org/ abs/1912.13466 (2019).

8. Katsouleas, T. Nature 431, 515-516 (2004)

9. Boscolo, M., Delahaye, J.-P. \& Palmer, M. Rev. Accel. Sci. Technol. 10, 189-214 (2019).

Climate science

\title{
Early models successfully predicted global warming
}

\author{
Jennifer E. Kay
}

Climate models published between 1970 and 2007 provided

accurate forecasts of subsequently observed global surface

warming. This finding shows the value of using global

observations to vet climate models as the planet warms.

Climate models are equations that describe climatically relevant processes and are solved on supercomputers. In addition to being invaluable tools for testing scientific hypotheses, these models have long provided societally important forecasts. The first climate models to numerically describe an evolving and interacting atmosphere, ocean and land surface on a grid covering the entire Earth date back to the 1970s (for example, refs 1-3). Since then, the planet's surface has warmed, in large part because of increased emissions of greenhouse gases. Writing in Geophysical Research Letters, Hausfather et al. ${ }^{4}$ retrospectively assessed the forecasting skill of climate models published between 1970 and 2007. Their results show that the physics in these early models was accurate in predicting subsequently observed global surface warming.

A key point emphasized by the authors is that the forecasting ability of climate models is limited by unknowable future climate drivers. Many major drivers, such as increased concentrations of carbon dioxide in the atmosphere caused by the burning of fossil fuels, result from human activities and decisions. Early climate modellers included estimates for future climate drivers in their forecasts. However, they could not know, for example, how the world would industrialize or the associated

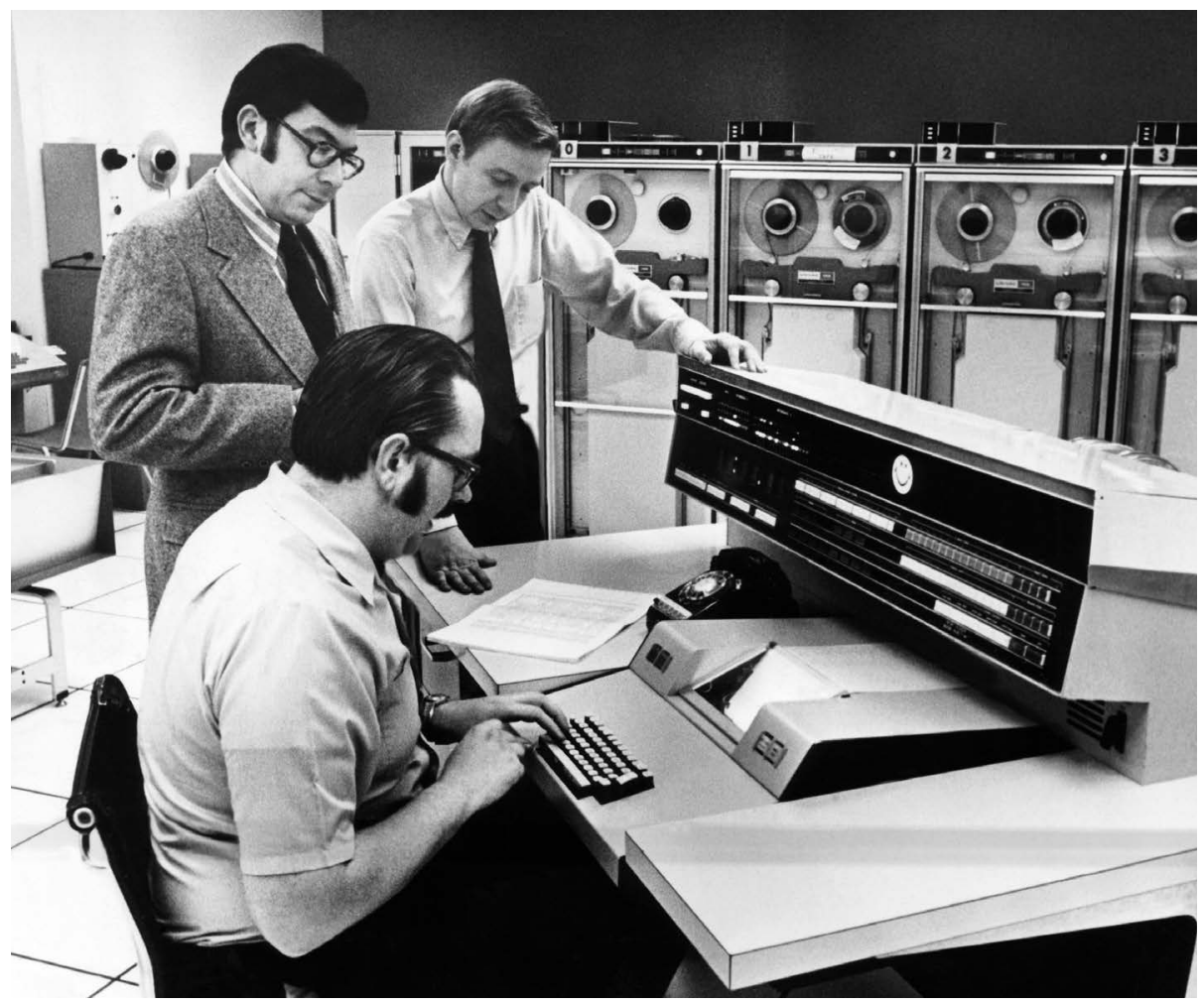

Figure 1 | A Univac 1108 computer, from 1972. Hausfather et al. ${ }^{4}$ demonstrate that climate models published over the past five decades accurately predicted subsequently observed changes in Earth's global mean surface temperature. These models include ones reported in the 1970s that used supercomputers, such as the Univac 1108, that had extremely limited power relative to those used today. 
emissions of $\mathrm{CO}_{2}$ that would result.

Hausfather and colleagues developed a method for evaluating the forecasts of early climate models without penalizing the models for their inaccurate estimates of unknowable future climate drivers. The authors examined 17 projections of global mean surface temperature (GMST) from 14 models. Before applying their method, they found that 10 projections were consistent with observations. But when inaccuracies in the estimates of climate drivers were taken into account, the authors discovered that 14 projections agreed with the data. Of the three that did not, two predicted higher-than-observed surface warming and one predicted lower-than-observed warming.

Developing credible climate models through an understanding of climatically relevant processes, observations and well-formulated equations is a considerable scientific and computational challenge. The equations that describe climate are complex and require substantial computing power to solve. As a result, climate models have always been run on the fastest supercomputers available. It is especially impressive that the earliest models assessed by Hausfather et al. produced accurate GMST forecasts, given the extremely limited computing power available then compared with that used today (Fig. 1).

Although the authors' findings show that climate models can accurately predict GMST, these forecasts are insufficient for understanding and preparing for the effects of ongoing climate change. For instance, regional climate change is especially subject to unpredictable climate variability, which greatly limits forecasting potential - even on decadal timescales when the climate drivers are known ${ }^{5}$. Moreover, on the basis of GMST forecasts alone, it is hard to predict, for example: to what extent sea level will rise; how ocean acidification caused by uptake of atmospheric $\mathrm{CO}_{2}$ will influence marine ecosystems; and the frequency and magnitude of future fires, droughts and floods.

Scientists will have to continue to improve climate modelling and to increase their understanding of the effects of climate change, while keeping in mind the tension between the need for increased model resolution, greater representation of climatically relevant processes, and more simulations to characterize unpredictable climate variability. The successful forecasting of GMST by early climate models is impressive, but leaves much work to be done - as scientists, policymakers and stakeholders are all well aware.

Numerical models based on scientific equations describing the atmosphere are used daily to make decisions that save lives and money. As the climate continues to change owing largely to human activities, scientists need to use, improve and communicate the value of numerical models and the equations and knowledge that underlie them. Hausfather and colleagues' work demonstrates that the physics in climate models has been providing accurate forecasts of GMST under increasing amounts of atmospheric $\mathrm{CO}_{2}$ for decades. Such predictions are useful for estimating the maximum amount of $\mathrm{CO}_{2}$ that can be released into the atmosphere over time to keep surface warming to a specified level.

Crucially, the authors' results also show that a major source of uncertainty in GMST forecasts comes from climate drivers. And, of these drivers, it is emissions of greenhouse gases from human activity that will largely determine future surface warming. The findings indicate the usefulness of climate-model predictions of GMST in response to increasing greenhouse-gas emissions, despite unknowable future climate drivers. But scientists must

also continue to develop climate models in concert with everything else available to them, to plan for a changed climate that requires much more than forecasts of surface warming.

Jennifer E. Kay is in the Department of Atmospheric and Oceanic Sciences and at the Cooperative Institute for Research in Environmental Sciences, University of Colorado at Boulder, Boulder, Colorado 80309, USA.

e-mail: jennifer.e.kay@colorado.edu

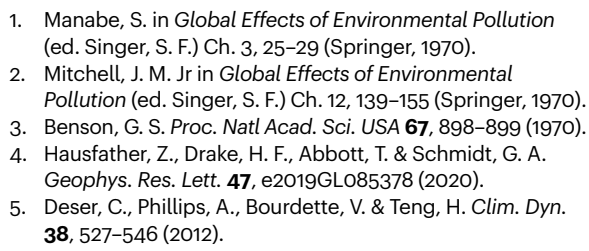

\title{
Cancer
}

\section{Brain tumours manipulate neighbouring synapses}

\author{
Nicola J. Allen
}

The growth of a brain tumour can be affected by the activity of its neighbouring neurons. The finding that such tumours send signals that boost connections between these neurons reveals a pathway that drives cancer growth. See p.166

A type of non-neuronal brain cell called a glial cell can give rise to a lethal cancer called glioblastoma ${ }^{1}$. Half of the cells in the human brain are glial cells, which normally act to support the function and communication of neurons ${ }^{2}$. Yet despite decades of research, there are no existing treatments for glioblastoma that substantially increase the survival time of people with such tumours. On page 166, Yu et al. ${ }^{3}$ report their analysis of the effects on the brain of certain glioblastoma-associated mutations. These insights might open up new strategies for anticancer research.

DNA sequencing of cancers has identified many tumour-associated mutations. However, it is a challenge to determine which of these mutations have a causal role in tumour development and growth, and which have no effect. Moreover, some mutations can be context dependent, such that the same mutation might have differing effects depending on the type of tumour and its microenvironment. It is hard to predict whether different mutations (variants) of the same gene will lead to the same outcome in different tumour types.

$\mathrm{Yu}$ and colleagues tackled these issues by studying the RTK-RAS-PI3K signalling pathway in glioblastoma. This pathway is altered in $90 \%$ of glioblastomas ${ }^{4,5}$, and mutations in it boost cell division and tumour growth. The authors focused on mutations in the gene encoding the enzyme PIK3CA - a pathway component that is often abnormal in human glioblastomas.

The authors generated mouse models of glioblastoma, and mutated genes in the RTKRAS-PI3K pathway using the gene-editing tool CRISPR-Cas9, which resulted in tumour growth. Yu et al. then engineered animals to express PIK3CA variants that are found in human glioblastomas (Fig. 1). This revealed that many of the tested variants made tumours more aggressive and rapidly lethal. This was the case both for known variants and for others that had not previously been associated with a role in glioblastoma.

Yu and colleagues studied whether alterations in the enzymatic activity of PIK3CA variants might explain how they accelerate glioblastoma progression. Surprisingly, the activity of PIK3CA was not always linked to an effect on tumour growth - some variants strongly increased enzyme activity, whereas others had a much milder effect. To investigate other mechanisms that might explain the effect 